\title{
Caracterização e influência dos fatores ambientais nas assembléias de peixes de riachos em duas microbacias urbanas, Alto Rio Paraná
}

\author{
Thiago Rota Alves Felipe ${ }^{1}$ \& Yzel Rondon Súarez ${ }^{1,2}$ \\ ${ }^{1}$ Laboratório de Ecologia, Centro Integrado de Análise e Monitoramento Ambiental, \\ Universidade Estadual de Mato Grosso do Sul - UEMS, \\ Rod. Dourados-Itahum, Km 12, CEP 79804-970, Dourados, MS, Brasil \\ ${ }^{2}$ Autor para correspondência: Yzel Rondon Súarez,e-mail: yzel@uems.br
}

FELIPE, T.R.A. \& SÚAREZ, Y.R. Characterization and influence of environmental factors on stream fish assemblages in two small urban sub-basins, Upper Paraná River. Biota Neotrop. 10(2): http://www.biotaneotropica.org.br/v10n2/en/abstract?article+bn03810022010.

\begin{abstract}
To quantify the influence of environmental descriptors on fish diversity and abundance in two small urban sub-basins in the Upper Paraná basin, we carried out two sampling series in the dry season (June and July) and two in the wet season (November and December), by means of a sieve $(80 \times 120 \mathrm{~cm})$ and a standardized effort (20 throws) per site. We collected 1,667 fish belonging to 20 species. Serrapinnus notomelas was the most abundant species, followed by Oligosarcus pintoi. The variations in species richness and evenness were explained by water electrical conductivity, stream depth, and stream width. The Cluster Analysis results showed a clear differentiation among the sites, with significant spatial differences and no seasonal variation in species composition. The first two axes of the Canonical Correspondence Analysis (CCA) explained 55\% of the variation in species abundance, and the main determinants of species distribution were stream width and water conductivity and turbidity. Keywords: water quality, freshwater fish, urban streams, water contamination.
\end{abstract}

FELIPE, T.R.A. \& SÚAREZ, Y.R. Influência dos fatores ambientais nas comunidades de peixes de riachos em duas microbacias urbanas, Alto Rio Paraná. Biota Neotrop. 10(2): http://www.biotaneotropica.org.br/ v10n2/pt/abstract?article+bn03810022010.

Resumo: Com o objetivo de quantificar a influência das características ambientais sobre a diversidade e abundância de peixes em duas microbacias urbanas na bacia do Alto Rio Paraná, foram realizadas duas amostragens no período de seca (junho e julho) e duas no período de chuva (novembro e dezembro) utilizando uma peneira $(80 \times 120 \mathrm{~cm})$, com esforço padronizado em 20 lances por local. Foram coletados 1.667 indivíduos, distribuídos em 20 espécies. A espécie mais abundante foi Serrapinus notomelas, seguida de Oligosarcus pintoi. A variação na riqueza de espécies e na equitabilidade foram explicadas pela condutividade, profundidade e largura dos riachos. Os resultados da análise de agrupamento e da ANOSIM mostraram diferença espacial significativa e ausência de variação temporal na composição de espécies. Os dois primeiros eixos da Análise de Correspondência Canônica (ACC) explicaram 55\% da variação na abundância das espécies e os principais determinantes na distribuição das espécies foram a largura dos riachos, condutividade elétrica e turbidez da água.

Palavras-chave: qualidade da água, peixes de água doce, riachos urbanos, contaminação da água. 


\section{Introdução}

Muitas características dos habitat influenciam os padrões de composição e diversidade das comunidades de peixes, como os filtros ambientais (Poff 1997), a disponibilidade de recursos (Zaret \& Rand 1971) e a complexidade dos habitat (Gorman \& Karr 1978). A despeito das muitas teorias que predizem a organização das comunidades, nenhuma delas é precisa quando ocorre o declínio da qualidade dos habitat, uma vez que ocorre uma homogeneização das assembléias, que passam a ser dominadas por espécies generalistas.

Distúrbios pouco freqüentes e de baixa magnitude são suportados pelas comunidades biológicas e podem, inclusive, manter a elevada diversidade de espécies (Connell 1978). No entanto, a continuidade deste distúrbio pode eliminar espécies mais sensíveis, alterando a organização das comunidades. As alterações na estrutura dos habitat pelo desmatamento, na bacia hidrográfica, alteram a dinâmica trófica e podem alterar a diversidade e composição de espécies de peixes (Bojsen \& Barriga 2002, Johnson 2002, Cetra \& Petrere 2006, Ferreira \& Casatti 2006).

A ictiofauna de riachos da bacia do Alto Rio Paraná tem recebido maior atenção nos últimos anos, com aumento dos estudos sobre a composição de espécies (Castro et al. 2003, 2004, 2005, Langeani et al. 2007), a influência dos fatores ambientais sobre a distribuição das espécies (Abes \& Agostinho 2001, Pavanelli \& Caramaschi 2003, Súarez et al. 2007, Súarez \& Lima Júnior 2009) e a sua biologia (Casatti et al. 2006, Gomiero \& Braga 2008, Rondineli et al. 2009, Súarez et al. 2009). Estes estudos tem se intensificado dada a percepção da fragilidade deste grupo frente à degradação da vegetação marginal e das variações das características físicas e químicas da água.

Alguns estudos têm verificado alterações na diversidade e composição das assembléias de peixes como resultado da alteração na estrutura dos habitats pelo desmatamento e despejo de dejetos orgânicos e industriais na bacia do Alto Rio Paraná (Smith et al. 1997, Barrella \& Petrere 2003, Lima-Junior et al. 2006, Casatti et al. 2006a, b, Cunico et al. 2006, Ferreira \& Casatti 2006). Contudo, apesar da constatação de elevada quantidade de pesquisas nesta bacia, na porção oeste da mesma, drenada pelo rio Ivinhema, praticamente inexistem estudos quantificando o impacto da alteração dos habitat nas assembléias de peixes.

O presente estudo teve como objetivo definir quais e quantas espécies de peixes ocorrem nas microbacias dos riachos Paragem e Engano, bacia do rio Dourados, Alto rio Paraná, se a riqueza, equitabilidade e composição de espécies são influenciadas pelas variações nas características ambientais e temporais nestes riachos e quais variáveis ambientais são mais importantes na determinação da distribuição das espécies.

\section{Materiais e Métodos}

\section{1. Área de estudo}

As amostragens foram realizadas em seis locais, sendo três na microbacia do riacho Água Boa e três na microbacia do riacho Engano (Figura 1). Na microbacia do riacho Água Boa as amostragens foram realizadas em dois riachos de primeira ordem (Paragem 1 e 2) e em um riacho de segunda ordem (Paragem 3) localizado à jusante de um pequeno açude na área urbana da cidade de Dourados-MS, este com área aproximada de 2,2 ha e um trecho de corredeira logo abaixo do açude. Na microbacia do riacho Engano, foram selecionados dois riachos de primeira ordem (riacho Chico Viégas e Olho D’água) e de um de segunda ordem (riacho Engano), sendo este localizado à jusante dos dois primeiros. Os critérios para seleção dos locais amostrados foram a facilidade de acesso e no caso do riacho Paragem a presença de estações de tratamento de esgoto à jusante dos locais amostrados, que conduziram à completa ausência de peixes, verificada em amostragens piloto anteriores ao período estudado.

Os locais amostrados apresentam largura entre 0,63 e 2,98 m e profundidade entre 0,38 e $0,78 \mathrm{~m}$. Os riachos selecionados nas duas bacias diferem principalmente quanto à largura, condutividade elétrica da água e concentração de oxigênio dissolvido (OD), sendo que os riachos da microbacia do riacho Água Boa são mais estreitos (largura média $=0,91 \mathrm{~m}$ ) e apresentam menor concentração de oxigênio $(\mathrm{OD}$ médio $=55,3 \%$ ) quanto comparados aos riachos da microbacia do riacho Engano (OD médio $=75,6 \%$ e largura média $=2,1 \mathrm{~m}$ ). Por outro lado, os riachos amostrados na bacia do Água Boa apresentam maiores valores de condutividade elétrica (condutividade $=157,3 \mu \mathrm{S} . \mathrm{cm}^{-1}$ ) quando comparados aos do riacho Engano (condutividade $=45,0 \mu \mathrm{S} . \mathrm{cm}^{-1}$ ) $($ Tabela 1 ), sendo as diferenças na condutividade elétrica associadas à localização da microbacia do riacho Paragem, dentro da área urbana da cidade de Dourados, enquanto os riachos da microbacia do riacho Engano se localizam em uma área peri-urbana, com trechos em área rural. Os locais amostrados na micro-bacia do riacho Água Boa não apresentam mata ripária, sendo que os dois primeiros apresentam uma cobertura de gramíneas nas margens e o terceiro, apresenta gramíneas, mas em uma região com residências em suas margens. O riacho Paragem 1 (P1) se apresenta como um trecho de remanso, enquanto os riachos Paragem 2 (P2) e Paragem 3 (P3) apresentam pequenos trechos de corredeira à montante dos locais amostrados. Dos riachos amostrados na micro-bacia do riacho Engano, o riacho Olho D'água (E1) se apresenta desprovido de mata ripária, também com gramíneas nas margens, enquanto os riachos Chico Viégas (E2) e Engano (E3) apresentam mata ripária secundária, uma vez que os trechos amostrados se localizam em sítios no entorno da cidade. Apenas o córrego Olho D’água apresenta uma pequena área alagável à jusante do trecho amostrado.

Do ponto de vista climatológico a região estudada é caracterizada como mesotérmico úmido, com verões quentes e invernos secos. Dezembro é o mês com maior pluviosidade, com média de 180,2 mm enquanto julho é o mês mais seco, com média de 45,9 mm (Fietz \& Fisch, 2008), desta forma no mês mais chuvoso a precipitação é aproximadamente quatro vezes superior ao mês mais seco.

\section{Amostragens}

As amostragens foram realizadas em junho e julho (estação seca) e novembro e dezembro (estação chuvosa) de 2003. Os peixes foram amostrados utilizando uma peneira retangular de armação metálica e tela mosquiteiro ( $2 \mathrm{~mm}$ de abertura de malha) sendo realizados 20 lances por local. Todos os riachos foram amostrados no período diurno em um trecho de aproximadamente $100 \mathrm{~m}$, os exemplares coletados foram fixados em formol a $10 \%$ e preservados em álcool etílico $70 \%$ para posterior identificação e contagem. Para a identificação dos peixes foi utilizado o trabalho de Graça \& Pavanelli (2007).

Em cada amostragem foram medidas as seguintes variáveis ambientais: $\mathrm{pH}$, condutividade $\left(\mu \mathrm{S} . \mathrm{cm}^{-1}\right)$, temperatura da água $\left({ }^{\circ} \mathrm{C}\right)$ e oxigênio dissolvido (\% de saturação), utilizando uma sonda multifunção YSI modelo 556. Uma amostra de água, da porção central e sub-superficial do trecho amostrado, foi levada ao laboratório para medição da turbidez, utilizando um turbidímetro de bancada Adamo, modelo TB1000. A profundidade e a largura do local (m) foram obtidas em cinco locais, aproximadamente equidistantes, ao longo de cada trecho amostrado (100 m), com a utilização de uma haste de madeira graduada ou de uma trena. Para as análises foi utilizada a média aritmética para os valores de largura e profundidade. 


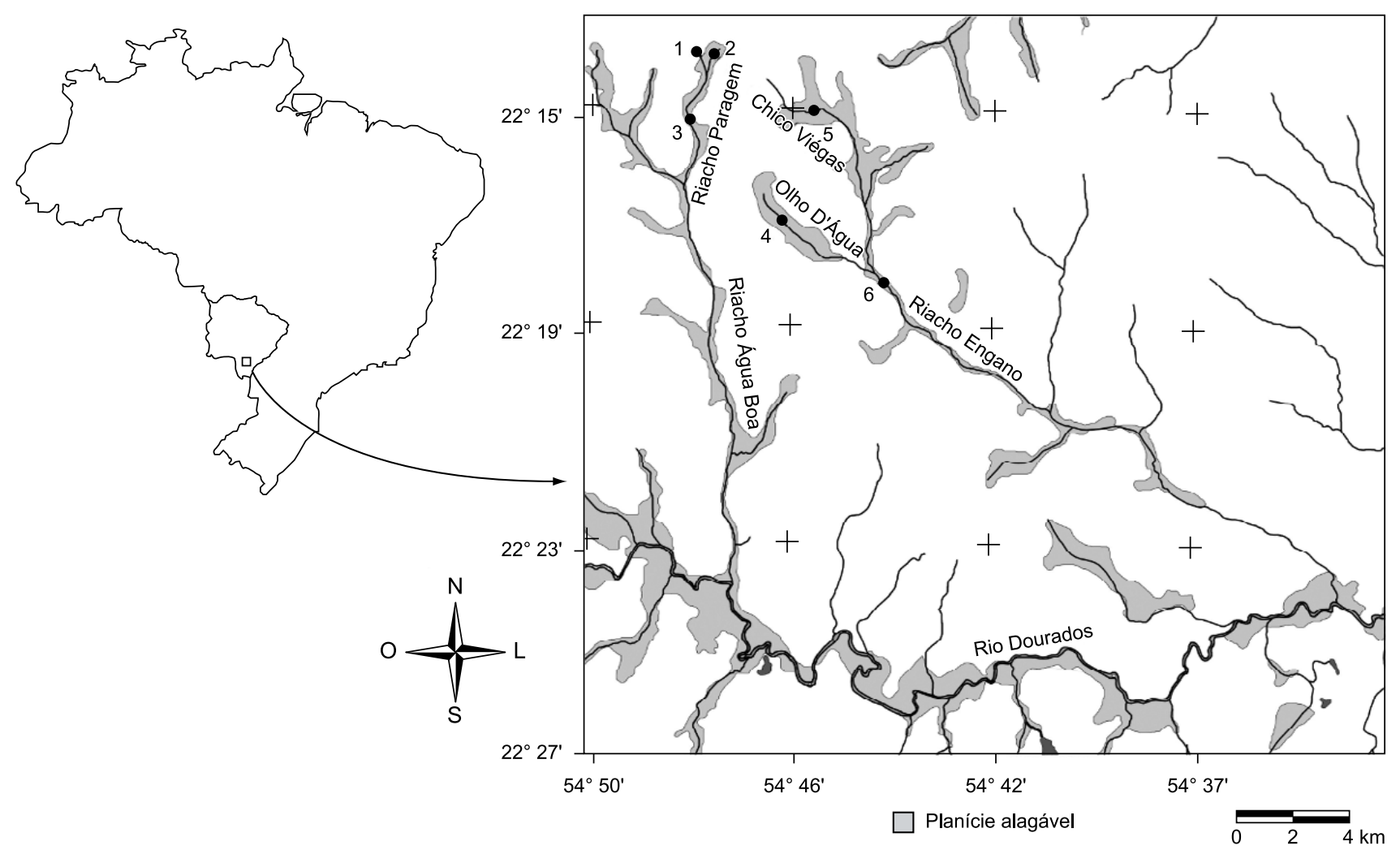

Figura 1. Localização dos riachos amostrados nas micro-bacias dos córregos Água Boa e Engano, Alto Rio Paraná. 1 = Riacho Paragem 1; 2 = Riacho Paragem 2; 3 = Riacho Paragem 3; 4 = Riacho Olho D'água; $5=$ Riacho Chico Viégas e $6=$ Riacho Engano.

Figure 1. Location of the streams sampled in the Água Boa and Engano sub-basins, Upper Paraná River. 1 = Paragem 1 stream; 2 = Paragem 2 stream; 3 = Paragem 3 stream; 4 = Olho D'água stream; 5 = Chico Viégas stream; and 6 = Engano stream.

Tabela 1. Valores médios de $\mathrm{pH}$, oxigênio dissolvido, condutividade elétrica da água, temperatura da água, turbidez, largura e profundidade dos riachos amostrados nas micro-bacias dos córregos Água Boa e Engano, Alto Rio Paraná.

Table 1. Mean values of $\mathrm{pH}$, dissolved oxygen, water conductivity, water temperature, turbidity, stream width and depth for sampled streams in Água Boa and Engano small basin, Upper Paraná River.

\begin{tabular}{lccccccc}
\hline \multicolumn{1}{c}{ Riachos } & $\mathbf{p H}$ & $\begin{array}{c}\text { Oxigênio dissolvido } \\
(\boldsymbol{\%} \text { de saturação) }\end{array}$ & $\begin{array}{c}\text { Condutividade } \\
\left(\boldsymbol{\mu} \mathbf{S} . \mathbf{c m}^{-\mathbf{1}}\right)\end{array}$ & $\begin{array}{c}\text { Temperatura } \\
\left({ }^{\circ} \mathbf{C}\right)\end{array}$ & $\begin{array}{c}\text { Turbidez } \\
(\mathbf{N T U})\end{array}$ & $\begin{array}{c}\text { Largura } \\
(\mathbf{m})\end{array}$ & $\begin{array}{c}\text { Profundidade } \\
(\mathbf{m})\end{array}$ \\
\hline Paragem 1 & 6,54 & 34,77 & 208 & 23,72 & 18,37 & 0,63 & 0,38 \\
Paragem 2 & 6,51 & 58,32 & 143 & 22,89 & 6,90 & 1,18 \\
Paragem 3 & 7,04 & 72,90 & 121 & 23,09 & 11,75 & 0,93 & 0,38 \\
Olho D'Água & 6,67 & 73,45 & 81 & 24,53 & 11,30 & 2,00 & 0,66 \\
Chico Viégas & 6,71 & 77,37 & 4 & 22,13 & 11,20 & 1,33 & 0,48 \\
Engano & 6,85 & 75,90 & 50 & 20,49 & 18,02 & 2,98 & 0,78 \\
\hline
\end{tabular}

\section{Análise dos dados}

A riqueza total, bem como seu intervalo de confiança, nos locais amostrados foi estimada por meio do método Jacknife (Heltshe \& Forrester 1983). Este método foi selecionado devido à sua robustez quando aplicado a amostras pequenas (Hellmann \& Fowler 1999).

Procurando verificar a influência dos descritores ambientais sobre a riqueza e equitabilidade das comunidades estudadas foi utilizada uma análise de árvore de regressão, onde a riqueza e equitabilidade foram utilizadas como variáveis resposta e os descritores ambientais como variáveis explanatórias. A árvore de regressão é um método não paramétrico de particionamento da variável resposta, onde as amostras são divididas em grupos mutuamente exclusivos e procurando aumentar a homogeneidade em cada grupo resultante, sua interpretação é simples e o método independe da forma de influência da variável explanatória sobre a variável resposta (ex: linear, exponencial, logística, entre outras) (De'Ath \& Fabricius 2000).

A variação espacial e temporal na composição de espécies foi quantificada através de uma análise de agrupamento, utilizando os dados de abundância das espécies e o coeficiente de Morisita-Horn. Foi utilizado o método de ligação pela media não ponderada (UPGMA), indicado por diminuir a distorção da matriz original durante a construção do dendrograma (Valentin, 2000). O coeficiente de correlação cofenética foi utilizado como medida de fidelidade do dendrograma à matriz original (McGarigal et al. 2000). 
Foi utilizada uma análise de similaridade (ANOSIM) para verificar a existência de diferença espacial (entre locais) e temporal (estações seca e chuvosa) na composição de espécies de peixes nos riachos amostrados (Clarke 1993), assim é possível definir se os locais (variação espacial) ou as estações do ano (variação temporal) diferem quanto a composição de espécies.

Após a padronização das variáveis ambientais pela média da variável analisada (média $=0$ e desvio padrão $=1$ ), sua influência sobre a distribuição em abundância das principais espécies de peixes (nove espécies representando pelo menos 1\% do total de indivíduos coletados) foi quantificada através de uma análise de correspondência canônica (ACC).

A seleção das espécies mais abundantes foi adotada como um critério para minimizar os resíduos na análise, resultado da ocorrência de espécies acidentais nas assembléias analisadas. Este é considerado um dos melhores métodos para análise de gradiente direto em ecologia de comunidades (Ter Braak 1986; Rodriguez \& Lewis 1997). A significância da influência das variáveis ambientais foi verificada através da função "envfit", que após a obtenção de um valor de $\mathrm{r}^{2}$ para as variáveis ambientais utiliza um processo de randomização para definir a significância destas variáveis (999 permutações) para todos os eixos em conjunto.

Todas as análises foram realizadas na plataforma $\mathrm{R}$ ( $\mathrm{R}$ Development Core Team 2005), sendo que a árvore de regressão foi realizada através do software rpart (Recursive partitioning and regression trees) e a análise de agrupamento, ACC e ANOSIM foram realizadas no software vegan (Oksanen et al. 2006).

\section{Resultados}

Foram coletados 1667 indivíduos distribuídos em 20 espécies. A espécie mais abundante foi Serrapinnus notomelas (Eigenmann) ( $\mathrm{n}=632$ indivíduos), seguido por Oligosarcus pintoi (Campos) $(\mathrm{n}=252)$ e Bryconamericus stramineus $($ Eigenmann $)(\mathrm{n}=214)$ (Tabela 2). A riqueza total estimada para os riachos amostrados foi de 25 , com intervalo de confiança $(\alpha=0,05)$ entre 20 e 30 espécies.

O riacho Olho D'água (E2) foi o que apresentou o maior número de espécies (12 espécies), seguida pelo riacho Paragem 3 (P3 = 11 espécies). Em dois locais da microbacia do riacho Água Boa (P2 e P3) O. pintoi foi a espécie mais abundante, sendo que Poecilia reticulata foi a segunda mais abundante nestes locais e a única a ocorrer no local Paragem 1 (P1). Na microbacia do riacho Engano, cada riacho apresentou uma espécie dominante diferente, sendo que $S$. notomelas foi a espécie dominante no riacho Olho D'água (E1), Astyanax altiparanae dominou no riacho Chico Viégas (E2) e B. stramineus foi a espécie dominante no riacho Engano (E3).

Os resultados da análise de árvore de regressão permitiram identificar a condutividade elétrica da água como o principal preditor da riqueza de espécies e equitabilidade, seguido da profundidade do riacho para a riqueza e pela largura do riacho para a equitabilidade. Os resultados mostraram ainda que $56,8 \%$ da variação na riqueza de espécies e $53 \%$ na variação na equitabilidade foram explicadas pelas variáveis selecionadas. Desta forma, riachos com condutividade elétrica da água igual ou superior a $169 \mu{\mathrm{S} . \mathrm{cm}^{-1}}_{\text {apresentam baixa }}$ riqueza de espécies (média $=1,6$ espécies) e baixa equitabilidade (média $=0,17$ ). Por outro lado, quando a condutividade oscilou entre 102 e $169 \mu \mathrm{S} . \mathrm{cm}^{-1}$ foi observada a maior riqueza de espécies (média $=6,7$ ). Com a condutividade menor que $169 \mu \mathrm{S} . \mathrm{cm}^{-1}$ e com largura inferior a $1,3 \mathrm{~m}$ foi constatada a maior media de equitabilidade $(0,83)$ (Figura 2).

$\mathrm{O}$ resultado da análise de agrupamento permitiu visualizar a formação de dois grupos interpretáveis (A e B), sendo o grupo "A", composto basicamente pelos riachos Engano e Olho D’água, ainda se divide nos grupos A1 e A2, enquanto o grupo B é composto predominantemente pelas amostras do riacho Paragem e pelo riacho Chico Viégas (Figura 3), onde o riacho Chico Viégas (B1) se separa dos demais (B2). Das quatro amostragens realizadas no riacho Paragem 1 três se isolaram das demais, fato explicado pelo fato de nenhuma espécie de peixe ter sido coletada nestas amostras.

O resultado da análise de similaridade sugere diferenças significativas na composição de espécies entre os riachos amostrados (ANOSIM $\mathrm{r}=0,468$; $\mathrm{p}<0,001$ ) sendo que apenas os riachos Paragem 2 e Paragem 3 são considerados estatisticamente iguais. Contudo, não foi constatada diferença temporal na composição de espécies para as assembléias analisadas (ANOSIM $\mathrm{r}=-0,073$; $\mathrm{p}=0,980)$.

Os primeiros dois eixos da análise de correspondência canônica explicaram $55 \%$ da variação na distribuição em abundância das espécies de peixes. Os descritores ambientais, considerados como significativos na distribuição da ictiofauna, foram a largura do riacho, condutividade elétrica e a turbidez da água (Tabela 3). Os resultados da ACC sugerem ainda que nos riachos estudados B. stramineus mostrou-se em maior abundância em trechos mais largos, com elevada turbidez e menores valores de condutividade. Por outro lado, $P$. reticulata ocorreu em maior abundância em locais mais estreitos e com elevada condutividade elétrica. Um grupo formado por A. altiparanae, C. paranaense, $C$. aeneus, $O$. pintoi, $H$. ancistroides e Gymnotus sp. ocorreu em maior abundância em trechos mais estreitos e com menores valores de turbidez e com valores elevados de condutividade. Serrapinnus notomelas não apresentou associação com as variáveis ambientais utilizadas (Figura 4).

\section{Discussão}

Os estudos sobre o efeito da urbanização sobre as assembléias de peixes ainda é escasso no Brasil, contudo, Barrella \& Petrere Júnior (2003) e Smith et al., (2009) analisaram a influência antrópica principalmente em rios de grande porte em São Paulo (bacias do Tietê e Paranapanema) e Pompeu et al., (2005) o fizeram em Minas Gerais (Rio das Velhas); Por outro lado, Cunico et al., (2006) e Alexandre et al. (2009) analisaram o efeito da urbanização sobre as assembléias de peixes de riachos em Maringá (PR) e Piracicaba (SP), todos constatando alterações na diversidade e composição de espécies em função da degradação pelo despejo de esgoto doméstico e industrial.

Amostragens realizadas em riachos com pouca influência da urbanização na bacia do rio Ivinhema tem sugerido que a condutividade elétrica nestes riachos apresenta valores entre 4 e $50 \mu \mathrm{S} \mathrm{cm}^{-1} \mathrm{em}$ trechos de cabeceira, podendo atingir valores de aproximadamente $100 \mu \mathrm{S} . \mathrm{cm}^{-1}$ em trechos inferiores da bacia e sob a influência recente de chuvas (Valério et al., 2007; Suarez \& Lima-Júnior, 2009). No presente estudo as nascentes das microbacias analisadas encontram-se distantes cerca de $3 \mathrm{~km}$ entre si, contudo, a condutividade foi em média 3,5 vezes maior nos locais amostrados no riacho Paragem, o que associado às diferenças na integridade dos habitat de entorno permite sugerir que estas duas microbacias apresentam diferenças na pressão antrópica. Apesar da maior degradação ambiental nos locais amostrados na microbacia do riacho Água Boa estes apresentaram os maiores valores médios de equitabilidade, com exceção do ponto Paragem 1. Este resultado corrobora as conclusões de Lima-Júnior et al. (2006) que sugerem que a diversidade de espécies (que inclui a equitabilidade) pode não refletir as alterações nas assembléias de peixes em função da poluição e propõem que a composição de espécies também seja utilizada nestes estudos.

A variação na composição de espécies nos ambientes lóticos é explicada por uma combinação de fatores locais e regionais 
Tabela 2. Lista e abundância das espécies de peixes nos locais amostrados nas microbacias dos córregos Água Boa e Engano, Alto rio Paraná. P1 = Paragem 1, P2 = Paragem 2, P3 = Paragem 3, E1 = Riacho Olho D'água, E2 = Riacho Chico Viégas e E3 = Riacho Engano.

Table 2. List and abundance of fish species in sampled sites in Água Boa and Engano small basin, Upper Paraná Basin. P1 = Paragem 1, P2 = Paragem 2, P3 = Paragem 3, E1 = Olho D'água Stream, E2 = Chico Viégas Stream and E3 = Engano Stream.

Espécies

Ordem Characiformes

Família Characidae

Astyanax altiparanae Garutti \& Britski, 2000

Astyanax sp.

Bryconamericus stramineus Eigenmann, 1908

Oligosarcus pintoi Campos, 1945

Piabina argentea Reinhardt, 1866

Serrapinnus notomelas (Eigenmann, 1915)

Hemigrammus marginatus Ellis, 1911

Família Lebiasinidae

Pyrrhulina australis Eigenmann \& Kennedy, 1903

Família Erythrinidae

Hoplias malabaricus (Bloch, 1794)

Ordem Gymnotiformes

Família Gymnotidae

Gymnotus sp.

Ordem Siluriformes

Família Callichthyidae

Corydoras aeneus (Gill, 1858)

Família Loricariidae

Hypostomus ancistroides (Ihering, 1911)

Hypostomus sp. 1

Hypostomus sp. 2

Família Pimelodidae

Imparfinis mirini Haseman, 1911

Ordem Perciformes

Família Cichlidae

Cichlasoma paranaense Kullander, 1983

Crenicichla britskii Kullander, 1982

Ordem Cyprinodontiformes

Família Rivulidae

Rivulus pictus Costa, 1989

Família Poecilidae

Poecilia reticulata Peters, 1859

Ordem Synbranchiformes

Família Synbranchidae

Synbranchus marmoratus Bloch, 1795

Número de Indivíduos

Riqueza de espécies

Equitabilidade média

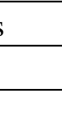

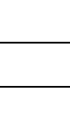

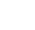

(Ricklefs 1987, Poff 1997) e nos locais amostrados as espécies dominantes são espécies comuns em toda a bacia do Alto Rio Paraná (Abes \& Agostinho 2001, Shibatta \& Cheida 2003, Langeani et al. 2005, Ferreira \& Casatti 2006), contudo, a elevada abundância de $P$. reticulata (e sua ocorrência exclusiva na microbacia do riacho Paragem) é um indicativo de uma resposta das assembléias de peixes

\begin{tabular}{ccccccc}
\multicolumn{3}{c}{ Água Boa } & & \multicolumn{3}{c}{ Engano } \\
\cline { 3 - 4 } \cline { 5 - 6 } P1 & P2 & P3 & & E1 & E2 & E3
\end{tabular}

$\begin{array}{rrrrrr}- & 44 & 15 & 16 & 48 & 13 \\ - & - & 1 & - & - & 8 \\ - & - & - & - & - & 214 \\ - & 110 & 120 & 22 & - & - \\ - & - & - & - & - & 1 \\ - & 71 & 7 & 466 & 5 & 83 \\ - & - & - & 1 & - & - \\ - & - & - & 14 & 1 & - \\ - & 1 & 8 & 2 & - & -\end{array}$

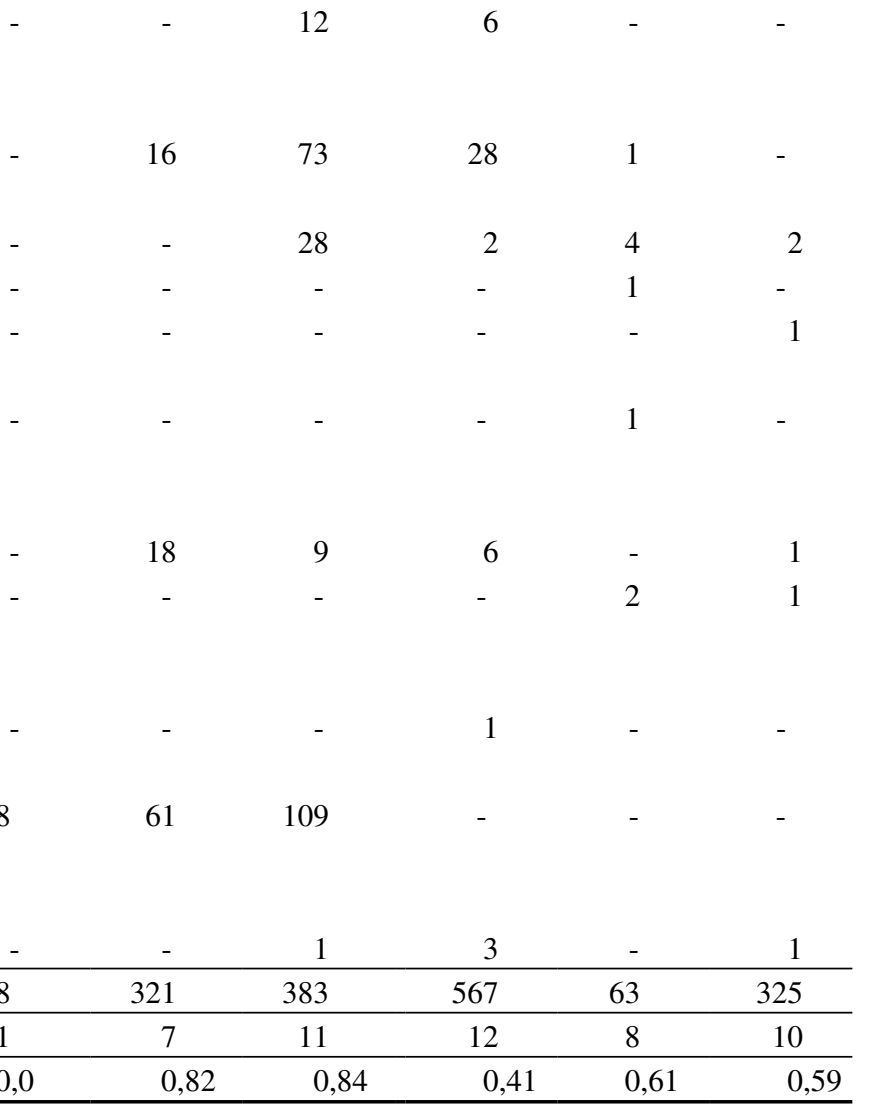

à degradação ambiental, devido à sua elevada tolerância à perda de qualidade ambiental (Cunico et al. 2006, Ferreira \& Casatti 2006, Pinto et al. 2006), de forma similar à observada para Phalloceros harpagos $=$ caudimaculatus $($ Barreto \& Uieda 1998, Bozzetti \& Schulz 2004, Vieira \& Shibatta 2007). Por outro lado, B. stramineus ocorreu exclusivamente no riacho Engano, o mais volumoso e com 
valores de turbidez elevados para os riachos estudados. Assim, a aparente associação de boa parte das espécies analisadas com os ambientes com maior condutividade pode ser o resultado da ocorrência exclusiva de $B$. stramineus em um local com características diferentes.

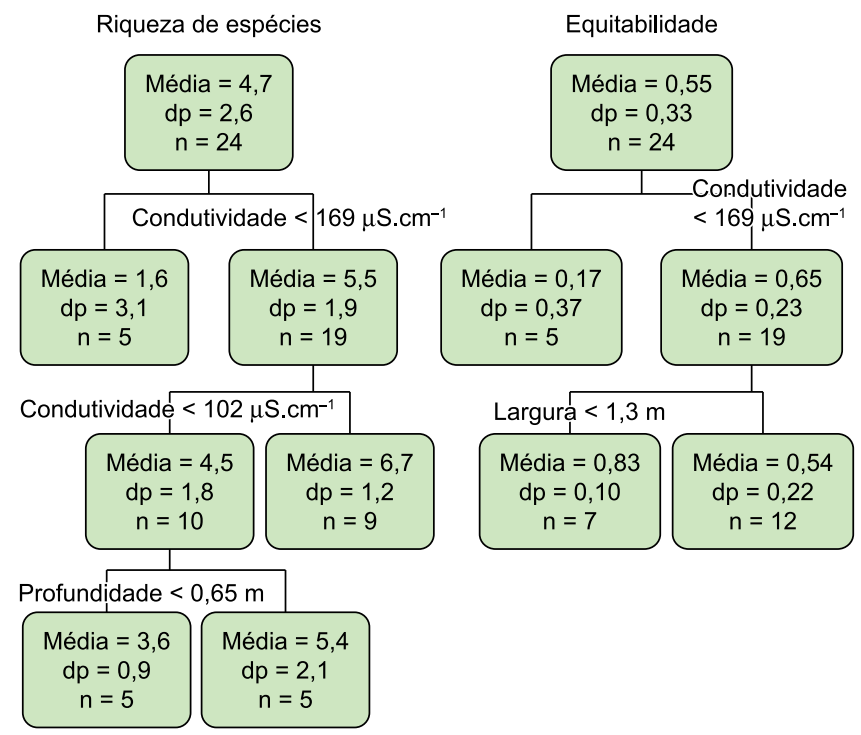

Figura 2. Árvore de regressão das características ambientais sobre a riqueza e equitabilidade nas assembléias de peixes estudadas nas micro-bacias dos córregos Água Boa e Engano, Alto Rio Paraná.

Figure 2. Regression tree of environmental characteristics on richness and evenness of fish assemblages in the Água Boa and Engano sub-basins, Upper Paraná River.
A condutividade elétrica da água e a profundidade e largura dos riachos foram os fatores que melhor explicaram a variação na diversidade de espécies (riqueza e equitabilidade) nos riachos amostrados. Estudos enfocando o impacto antrópico sobre as comunidades aquáticas (eg. Oliveira \& Bennemann 2004, Ferreira \& Casatti 2006, Cunico et al. 2006, Alexandre et al. 2009) usualmente encontram resultados similares, em que o aumento da degradação ambiental, aqui representada pela condutividade elétrica (resultado do despejo de resíduos orgânicos), leva a uma homogeneização das comunidades aquáticas, que muitas vezes passa pela perda de espécies nativas mais exigentes e pelo aumento da abundância de espécies tolerantes à perda de qualidade da água (eg. $P$. reticulata). No presente estudo foi constatado que os locais com maior condutividade apresentaram menores valores médios de riqueza, uma vez que, principalmente no ponto Paragem 1, apenas em uma das coletas foram encontrados peixes ( $P$. reticulata). Contudo, os locais Paragem 2 e Paragem 3 apresentam em comum maiores valores de correnteza, mantendo a concentração de oxigênio mais elevada nestes locais, assim não foi registrada hipóxia durante a amostragem, o que pode ter mantido a riqueza e equitabilidade mais elevada. Oliveira \& Bennemann (2004) estudando a alimentação e influência antrópica sobre a ictiofauna em uma micro-bacia na bacia do rio Tibagi (PR) constataram que $P$. reticulata e $P$. caudimaculatus (provavelmente $=P$. harpagos) predominaram em locais com maior nível de degradação ambiental, que foi representada tanto pelo desmatamento das margens, quanto pelo despejo de efluentes industriais nos riachos amostrados. Desta forma, os nossos dados corroboram o potencial uso de $P$. reticulata como indicador de perda de qualidade ambiental e sua ocorrência exclusiva na micro-bacia do riacho Água Boa reforça a sua maior degradação quando comparada à micro-bacia do riacho Engano.

Quanto às características físicas dos habitats, a teoria ecológica sugere que a diversidade de nichos ecológicos seja um dos principais responsáveis pela diversidade de espécies. De forma complementar,

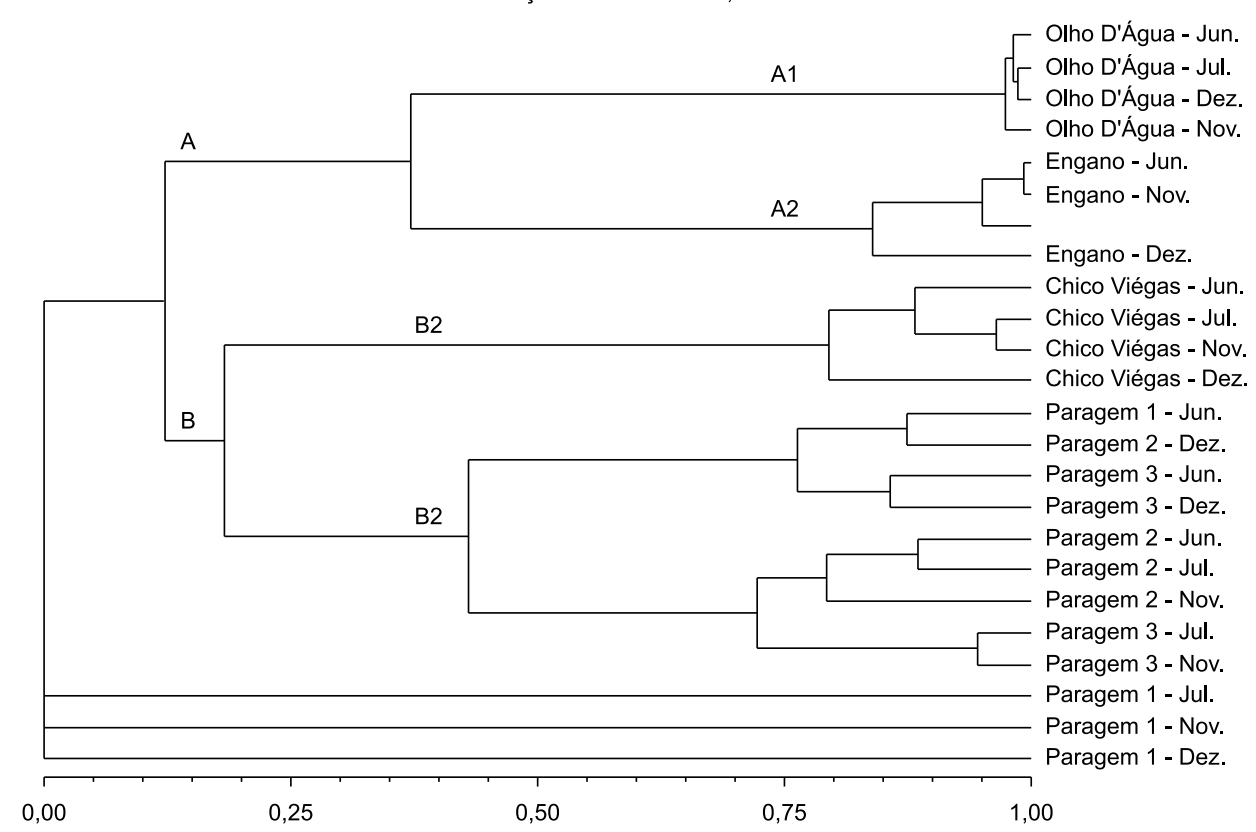

Figura 3. Dendrograma de similaridade na abundância das espécies (Índice de Morisita-Horn) para os locais amostrados nas micro-bacias dos córregos Água Boa e Engano, Alto rio Paraná.

Figure 3. Similarity dendrogram of species abundance (Morisita-Horn Index) for sites sampled in the Água Boa and Engano sub-basins, Upper Paraná River. 
Tabela 3. Resultado da análise de correspondência canônica (CCA) para as assembléias de peixes e os descritores ambientais nas microbacias dos córregos Água Boa e Engano, Alto Rio Paraná. ns = não significativo; * = significativo a $\alpha=0,05 ; * * *=$ significativo a $\alpha=0,001$.

Table 3. Results of canonical correspondence analysis (CCA) for fish assemblages and environmental descriptors in the Água Boa and Engano smallbasin, Upper Paraná River. ns = non-significant; * = significant at $\alpha=0.05$; $* * *=$ significant at $\alpha=0.001$.

\begin{tabular}{lccc}
\hline Descritores ambientais & Eixo 1 & Eixo 2 & $\mathbf{r}^{\mathbf{2}}$ \\
\hline $\mathrm{pH}$ & $-0,20$ & 0,98 & $0,07 \mathrm{~ns}$ \\
Oxigênio dissolvido (\%) & 0,91 & $-0,41$ & $0,07 \mathrm{~ns}$ \\
Condutividade & $-1,00$ & 0,06 & $0,46 *$ \\
Temperatura da água & $-0,58$ & $-0,81$ & $0,34 \mathrm{~ns}$ \\
Turbidez da água & 0,96 & 0,28 & $0,39 *$ \\
Largura do riacho & 0,99 & $-0,12$ & $0,96 * * *$ \\
Profundidade do riacho & 0,90 & $-0,44$ & $0,28 \mathrm{~ns}$ \\
\hline Variação explicada (\%) & 36 & 19 & - \\
\hline
\end{tabular}

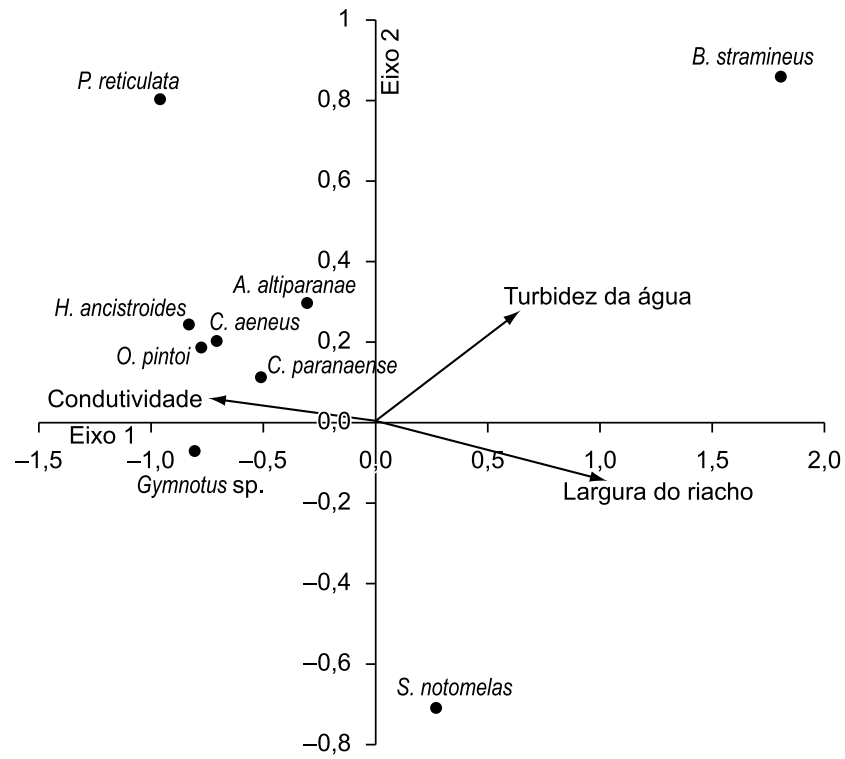

Figura 4. Diagrama de dispersão as análise de correspondência canônica (ACC) para as assembléias de peixes nas micro-bacias dos córregos Água Boa e Engano, Alto rio Paraná.

Figure 4. Scatterplot of canonical correspondence analysis (CCA) for fish assemblages in the Água Boa and Engano sub-basins, Upper Paraná River.

o volume de um rio é considerado um bom descritor da diversidade de habitats (Gorman \& Karr 1978), desta forma a importância da profundidade e largura dos riachos para a riqueza e equitabilidade nos riachos amostrados parece reforçar esta idéia, ainda que os resultados aqui apresentados demonstrem que a qualidade da água (aqui representada pela diferença na condutividade) assuma o papel de determinante primário da diversidade de espécies para os riachos aqui estudados.

A concordância entre os resultados da análise de agrupamento e a análise de similaridade evidenciam a elevada variação espacial e baixa variação temporal na composição de espécies, resultado similar ao observado em outros estudos (Grossman et al. 1998, Bührnheim \& Cox-Fernandes 2001, Valério et al. 2007, Súarez \& Petrere Júnior 2005, Súarez \& Lima-Junior 2009, Rocha et al. 2009). Grossman et al. (1998) comentam que, a despeito da variação temporal estar associada à migração dos peixes vindos de trechos inferiores da bacia, esta migração não altera o uso do espaço pelas espécies residentes e não altera significativamente a estrutura das comunidades de peixes; de forma complementar, Súarez \& Petrere Júnior (2005) sugeriram que a variação temporal é pequena em riachos de cabeceira. Então, o efeito restritivo das características hidrológicas parece minimizar a variação temporal na composição de espécies.

A grande importância da largura dos riachos na determinação da abundância das espécies pode ser parcialmente atribuída à ocorrência exclusiva de $B$. stramineus e Astyanax sp. no riacho Engano, enquanto $P$. reticulata predominou no riacho Paragem 1 (com menor largura), o que pode ser visualizado na Figura 4.

Concluindo, o presente trabalho sugere que o número de espécies e equitabilidade nos riachos amostrados foram determinados primariamente pela diferença na integridade dos riachos, uma vez que a condutividade foi o principal determinante da diversidade de espécies. Constatou-se também que estes riachos não apresentaram variação sazonal significativa na composição de espécies, mas apresentaram diferenças claras entre riachos amostrados. Os riachos mais afetados pela interferência antrópica apresentaram redução da riqueza e equitabilidade, assim como predomínio de espécies mais tolerantes à perturbação ambiental.

\section{Agradecimentos}

A UEMS pelo apoio logístico e pela bolsa de Iniciação Científica (PIBIC/UEMS) concedida ao primeiro autor. Aos revisores anônimos pelas sugestões que levaram à melhoria do manuscrito.

\section{Referências Bibliográficas}

ABES, S.S. \& AGOSTINHO, A.A. 2001. Spatial patterns in fish distributions and structure of the icthyocenosis in the Água Nanci stream, upper Paraná river basin, Brazil. Hydrobiol. 445(1-3):217-227.

ALEXANDRE, C.V., ESTEVES, K.E. \& MELO, M.A.M.M. 2009. Analysis of fish communities along a rural-urban gradient in a neotropical stream (Piracicaba river basin, São Paulo, Brazil). Hydrobiol. 641(1):97-114.

BARRELLA, W. \& PETRERE-Jr., M. 2003. Fish community alterations due to pollution and damming in Tietê and Paranapanema rivers (Brazil). River Res. Appl. 19(1):59-76.

BARRETO, M.G. \& UIEDA, V.S. 1998. Influence of the abiotic factors on the ichthyofauna composition in different orders stretches of Capivara River, São Paulo State, Brazil. Verh. Internat. Verein. Limnol. 26:2180-2183.

BOJSEN, B.H. \& BARRIGA, R. 2002. Effect of deforestation on fish community structure in Ecuadorian Amazon streams. Freshw. Biol. 47(11):2246-2260.

BOZZETTI, M. \& SCHULZ, U.H. 2004. An index of biotic integrity based on fish assemblages for subtropical streams in southern Brazil. Hydrobiol. 539(1-3):133-144.

BÜHRNHEIM, C.M. \& COX-FERNANDES, C. 2001. Low seasonal variation on fish assemblages in Amazonian rain forest stream. Ichthyol. Explor. Freshwaters 12(1):65-78.

CASATTI, L., CARVALHO, F.R., VERONEZI-Jr., J.L. \& LACERDA, D.R. 2006. Reproductive biology of the Neotropical superfetaceous Pamphorichthys hollandi (Cyprinodontiformes: Poeciliidae). Ichthyol. Explor. Freshwaters 17(1):59-64.

CASATTI, L., LANGEANI, F. \& FERREIRA, C.P. 2006a. Effects of the physical habitat degradation on the stream fish assemblage structure in a pasture region. Environ. Manage. 38(6):974-982.

CASATTI, L., LANGEANI, F., SILVA, A.M. \& CASTRO, R.M.C. 2006b. Stream fishes, water and habitat quality in a pasture dominated basin, southeastern Brazil. Braz. J. Biol. 66(2):681-696.

CASTRO, R.M.C., CASATTI, L., SANTOS, H.F., FERREIRA, K.M., RIBEIRO, A.C., BENINE, R.C., DARDIS, G.Z.P., MELO, A.L.A., ABREU, T.X., BOCKMANN, F.A., CARVALHO, M., GIBRAN, F.Z. \& 
LIMA, F.C.T. 2003. Estrutura e composição da ictiofauna de riachos do Rio Paranapanema, sudeste e sul do Brasil. Biota Neotrop. 3(1): <http:// www.biotaneotropica.org.br/v3n1/pt/abstract?article+BN01703012003> (último acesso em 09/12/2009).

CASTRO, R.M.C., CASATTI, L., SANTOS, H.F., MELO, A.L.A., MARTINS, L.S.F., FERREIRA, K.M., GIBRAN, F.Z., BENINE, R.C., CARVALHO, M., RIBEIRO, A.C., ABREU, T.X., BOCKMANN, F.A., DARDIS, G.Z.P., STOPIGLIA, R. \& LANGEANI, F. 2004. Estrutura e composição da ictiofauna de riachos da bacia do Rio Grande, no Estado de São Paulo, Sudeste do Brasil. Biota Neotrop. 4(1): <http://www.biotaneotropica. org.br/v4n1/pt/abstract?article+BN0170402004> (último acesso em 09/12/2009).

CASTRO, R.M.C., CASATTI, L., SANTOS, H.F., VARI, R.P., MELO, A.L.A., MARTINS, L.S.F., ABREU, T.X., BENINE, R.C., GIBRAN, F.Z., RIBEIRO, A.C., BOCKMANN, F.A., CARVALHO, M., PELIÇÃO, G.Z., FERREIRA, K.M., STOPIGLIA, R. \& AKAMA, A. 2005. Structure and composition of the stream ichthyofauna of four tributary rivers of the upper Rio Paraná basin, Brazil. Ichthyol. Explor. Freshwaters 16(3):193-214.

CETRA, M. \& PETRERE Jr., M. 2006. Fish assemblage structure of the Corumbataí River Basin, São Paulo State, Brazil: Characterization and anthropogenic disturbances. Braz. J. Biol. 66(2A):431-439.

CLARKE, K.R. 1993. Non-parametric multivariate analysis of changes in community structure. Aust. J. Ecol. 18(1):117-143.

CONNELL, J.H. 1978. Diversity in tropical rain forest and coral reefs. Science 199(4335):1302-1310.

CUNICO, A.M., AGOSTINHO, A.A. \& LATINI, J.D. 2006. Influência da urbanização sobre as assembléias de peixes em três córregos de Maringá, Paraná. Rev. Bras. Zool. 23(4):1101-1110.

DE'ATH, G. \& FABRICIUS, K. E. 2000. Classification and regression trees: a powerful yet simple technique for ecological data analysis. Ecology 81(11):3178-3192.

FERREIRA, C.P. \& CASATTI, L. 2006. Integridade biótica de um córrego na bacia do Alto Rio Paraná avaliada por meio da comunidade de peixes. Biota Neotrop. 6(3): <http://www.biotaneotropica.org.br/v6n3/pt/ abstract?article+bn00306032006> (último acesso em 19/11/2009).

FIETZ, C.R. \& FISCH, G.F. 2008. O clima da região de Dourados, MS. 2 ed. Embrapa Agropecuária Oeste, Dourados, 32p. (Série Documentos, 92).

GOMIERO, L.M. \& BRAGA, F.M.S. 2008. Feeding habits of the ichthyofauna in a protected area in the state of São Paulo, southeastern Brazil. Biota Neotrop. 8(1): <http://www.biotaneotropica.org.br/v8n1/en/ abstract?article+bn00608012008> (último acesso em 09/12/2009).

GORMAN, O.T. \& KARR, J.R. 1978. Habitat structure and stream fish communities. Ecology 59(3):507-515.

GRAÇA, W.J. \& PAVANELLI, C.S. 2007. Peixes da planície de inundação do alto rio Paraná e áreas adjacentes. EDUEM, Maringá, 241p.

GROSSMAN, G.D. \& RATAJCZAK, R.E.Jr. 1998. Long-term patterns of microhabitat use by fish in a southern Appalachian stream from 1983 to 1992: effects of hydrologic period, season and fish length. Ecol. Freshw. Fish 7:108-131.

HELLMANN, J.J. \& FOWLER, G.W. 1999. Bias, precision and accuracy of four measures of species richness. Ecol. Appl. 9(3):824-834.

HELTSHE, J.F. \& FORRESTER, N.E. 1983. The jackknife estimate of species richness. Biometrics 39:1-11.

JOHNSON, W.C. 2002. Riparian vegetation diversity along regulated rivers: contribution of novel and relict habitats. Freshw. Biol. 47:749-759.

LANGEANI, F., CASATTI, L., GAMEIRO, H.S., CARMO, A.B. \& ROSSA-FERES, D.C. 2005. Riffle and pool fish communities in a large stream of southeastern Brazil. Neotrop. Ichthyol. 3(2):305-311.

LANGEANI, F., CASTRO, R.M.C., OYAKAWA, O.T., SHIBATTA, O.A., PAVANELLI, C.S. \& CASATTI, L. 2007. Diversidade da ictiofauna do Alto Rio Paraná: composição atual e perspectivas futuras. Biota Neotrop. 7(3): <http://www.biotaneotropica.org.br/v7n3/pt/ abstract?article+bn03407032007> (último acesso em 09/12/2009).
LIMA-Jr., S.E., CARBONE, I.B. \& GOITEN, R. 2006. Fish assemblage structure and aquatic pollution in a Brazilian stream: some limitations of diversity indices and models for environmental impact studies. Ecol. Freshw. Fish 15(3):284-290.

McGARIGAL, K., CUSHMAN, S. \& STAFFORD, S. 2000. Multivariate statistics for wildlife and ecology research. Springer-Verlag, New York, 283p.

OKSANEN, J., KINDT, R., LEGENDRE, P. \& O'HARA, B. 2006. Community Ecology Package: Vegan. Version. 1.8.1. <http://cc.oulu. fi/ jarioksa/> (último acesso em 09/12/2009).

OLIVEIRA, D.C. \& BENNEMANN, S.T. 2004. Ictiofauna, recursos alimentares e relações com as interferências antrópicas em um riacho urbano no Sul do Brasil. Biota Neotrop. 5(1): <http://www. biotaneotropica.org.br/v5n1/pt/abstract?article+BN02905012005> (último acesso em 09/12/2009).

PAVANELLI, C.S. \& CARAMASCHI, E.P. 2003. Temporal and spatial distribution of the ichthyofauna in two streams of the upper Rio Paraná Basin. Braz. Arch. Biol. Technol. 46:271-280.

PINTO, B.C.T., PEIXOTO, M.G. \& ARAÚJO, F.G. 2006. Effects of the proximity from an industrial plant on fish assemblages in the rio Paraíba do Sul, southeastern Brazil. Neotrop. Ichthyol. 4(2):269-278.

POFF, N.L. 1997. Landscape filters and species traits: towards mechanistic understanding and prediction in stream ecology. J. N. Am. Benthol. Soc. 16(2):391-409.

POMPEU, P.S., ALVES, C.B. \& CALLISTO, M. 2005. The effects of urbanization on biodiversity and water quality in the Rio das Velhas basin, Brazil. Am. Fish. Soc. Symp. 47:11-22.

R Development Core Team. 2005. R: A language and environment for statistical computing. R Foundation for Statistical Computing, Vienna.

RICKLEFS, R.E. 1987. Community diversity: relative roles of local and regional processes. Science 235(4785):167-171.

ROCHA, F.C., CASATTI, L. \& PEREIRA, D.C. 2009. Structure and feeding of a stream fish assemblage in Southeastern Brazil: evidence of low seasonal influences. Acta Limnol. Bras. 21(1):123-134.

RODRÍGUEZ, M.A. \& LEWIS, W.M. 1997. Structure of fish assemblages along environmental gradients in floodplain lakes of the Orinoco River. Ecol. Monogr. 67(1):109-128.

RONDINELI, G.R., CARMASSI, A.L. \& BRAGA, F.M.S. 2009. Population biology of Trichomycterus sp. (Siluriformes, Trichomycteridae) in Passa Cinco stream, Corumbataí river sub-basin, São Paulo state, southeastern Brazil. Braz. J. Biol. 69(3):925-934.

SHIBATTA, O.A. \& CHEIDA, C.C. 2003. Composição em tamanho de peixes (Actinopterygii, Teleostei) de ribeirões da bacia do rio Tibagi, Paraná, Brasil. Rev. Bras. Zool. 20(3):469-473.

SMITH, W. S., PETRERE Jr., M. \& BARRELLA, W. 2009. The fish community of the Sorocaba River Basin in different habitats (State of São Paulo, Brazil). Braz. J. Biol. 69(4):1015-1025.

SMITH, W.S., BARRELLA, W. \& CETRA, M. 1997. Comunidade de peixes como indicadora de poluição ambiental. Rev. Bras. Ecol. 1(1):67-71.

SÚAREZ, Y.R. \& LIMA Jr., S.E. 2009. Variação espacial e temporal nas assembléias de peixes de riachos na bacia do rio Guiraí, Alto Rio Paraná. Biota Neotrop. 9(1): <http://www.biotaneotropica.org.br/v9n1/pt/ abstract?article+bn01709012009> (último acesso em 09/12/2009).

SÚAREZ, Y.R. \& PETRERE Jr., M. 2005. Organização das assembléias de peixes em riachos da bacia do rio Iguatemi, Mato Grosso do Sul. Acta Sci. Biol. Sci. 22(2):161-167.

SÚAREZ, Y.R., SILVA, J.P., VASCONCELOS, L.P. \& ANTONIALLI Jr., W.F. 2009. Ecology of Phallotorynus pankalos (Cyprinodontiformes, Poeciliidae) in a first-order stream of the upper Paraná Basin. Neotrop. Ichthyol. 7(1):49-54.

SÚAREZ, Y.R., VALÉRIO, S.B., TONDATO, K.K., XIMENES, L.Q.L. \& FELIPE, T.R.A. 2007. Determinantes ambientais da ocorrência de espécies de peixes em riachos de cabeceira da bacia do rio Ivinhema, Alto Rio Paraná. Acta Sci. Biol. Sci. 19(2):145-150. 
TER BRAAK, C.J.F. 1986. Canonical correspondence analysis: a new eigenvector technique for multivariate direct gradient analysis. Ecology 67(5):1167-1179.

VALÉRIO, S.B., SÚAREZ, Y.R., FELIPE, T.R.A., TONDATO, K.K. \& XIMENES, L.Q.L. 2007. Organization patterns of headwater-stream fish communities in the Upper Paraguay-Paraná basins. Hydrobiol. 583(1):241-250.

VIEIRA, D.B. \& SHIBATTA, O.A. 2007. Peixes como indicadores da qualidade ambiental do ribeirão Esperança, Município de Londrina, Paraná, Brasil. Biota Neotrop. 7(1): <http://www.biotaneotropica. org.br/v7n1/pt/abstract?article+bn01407012007> (último acesso em 19/11/2009).

ZARET, T.M. \& RAND, A.S. 1971. Competition in tropical stream fishes: support for the competitive exclusion principle. Ecology 52(2):336-342.

Recebido em 13/12/09 Versão reformulada recebida em 05/06/10 Publicado em 17/06/10 